\title{
Design of a Novel Passive Wireless Integrated SAW-Based Antenna Sensor for Structural Health Monitoring
}

\author{
Xuan Dai, Lili Fang $\mathbb{D}^{D}$, Chuanfang Zhang $\mathbb{D}$, and Houjun Sun \\ School of Information and Electronics, Beijing Institute of Technology, 100081, China \\ Correspondence should be addressed to Lili Fang; fanglili_bit@126.com
}

Received 9 November 2019; Accepted 24 December 2019; Published 13 January 2020

Academic Editor: Vincenzo Stornelli

Copyright @ 2020 Xuan Dai et al. This is an open access article distributed under the Creative Commons Attribution License, which permits unrestricted use, distribution, and reproduction in any medium, provided the original work is properly cited.

\begin{abstract}
A novel passive wireless integrated SAW-based antenna sensor for strain sensing is presented in this paper. A SAW delay line scheme is proposed for signal modulation, which could distinguish the backscattered data from environmental clutter in time domain. The theoretical relationship between the antenna resonance frequency shifts, the temperature, and the applied strain was established. A multiphysical coupled simulation process is proposed to improve the simulation accuracy. By comparing the phase shift of adjacent echoed data which is generated by SAW reflective grating, the temperature information could be extracted exactly and the effect of temperature fluctuation on the resonance frequency could be compensated. A more accurate passive (battery-free) wireless strain sensing could be provided by this proposed integrated antenna sensor than the previous proposed methods. Simulation and experimental results demonstrate the effectiveness of the sensor.
\end{abstract}

\section{Introduction}

In recent years, there has been an increasing interest in wireless sensing technologies for structural health monitoring (SHM) [1]. SHM systems are desired on all aircrafts for dynamic monitoring of structures; they are expected to improve aircraft safety, reduce maintenance frequency and costs, and extend the operational life cycle of the aircraft. SHM systems could also be applied to static monitoring application scenarios, such as modern wooden building monitoring, cultural heritages environmental monitoring, and long-span arch bridges [2-4]. Sensors have been developed to measure various structural responses and environmental conditions, including strain, vibration, acceleration, humidity, and temperature. In the measurements, strain sensors attempt to address the stress concentration point and detect crack development; this can be critical to ensure structural integrity. Several types of sensors have been investigated for SHM applications, including strain gauges, fiber optic sensors, and piezoelectric transducer $[5,6]$. However, these methods require lengthy cable connections and batteries for operation, which can significantly increase the total mass of the vehicle, installation time, and system cost.

Wireless sensing is a type of new SHM technology, which could help to greatly reduce installation time and system cost. A huge amount of work about wireless sensors and their applications in SHM has been done $[7,8]$; most of these technologies use an additional wireless transmitter, which could translate physical sensing signals to electromagnetic signals. However, the wireless transmitter still needs a battery supply, which has a limited life cycle. Such a scheme is not feasible for harsh environment applications. All of these problems could be solved if the sensor itself acts both as a physical quantity-sensing device and as a communication device. SAW sensors could act as passive wireless strain sensors with high sensitivity. However, SAW strain sensors have limited strain sensing range, which could not meet the requirement of some large strain range applications, such as wind turbine blades.

In a particular antenna, the resonance frequency is related to its electrical dimension. If bonding or embedding the antenna to a flexible structure, the relationship between 
the antenna's resonance frequency and strain can be used for stress/strain measurement. Several types of antenna sensors have been proposed for wireless sensing [9-12].

A patch antenna could be used for wireless strain sensing [13-15]; when applying strain on the patch antenna, the geometry and the current distribution of the patch antenna will be changed, as a result, the resonant frequency will change accordingly. Reflection coefficient $S_{11}$ is measured to determine deformation/strain values. However, the patch antenna sensor needs an additional signal modulation scheme to separate the interrogation signal from the backscattered sensing signals.

A frequency doubling technique could be used as an alternative way of signal modulation for antenna sensor [16]. By doubling the backscattered signal's frequency, unwanted environmental reflections are differentiated. However, frequency doubling is implemented by the Schottky diode, which might distort the backscattered signal. For this reason, the strain/stress sensing accuracy of this approach is limited.

RFID (radio frequency identification) antennas have been proposed for wireless strain sensing [17, 18]. An RFID antenna sensor contains an integrated RFID chip for signal modulation; the return signal from the RFID antenna sensor is modulated with a unique pattern to be separated from environmental noise. However, the commercially available RFID chips have limited operating frequency range such as $860-960 \mathrm{MHz}$; the size of the antenna is therefore limited. Since the strain sensitivity of the antenna sensor is directly related to the operating resonance frequency, the RFID antenna sensors have relatively low strain sensitivities.

Thermal effects can have significant effects on antenna sensor performance [19]. When temperature fluctuates, the dielectric constant of the substrate (such as glass microfiberreinforced PTFE substrate) which is used in printed antenna sensors will change accordingly. Since the resonance frequency is the function of both the dielectric constant and the electrical dimension, the antenna sensing accuracy will decrease with temperature variation.

The presented research realizes an integrated SAW-based patch antenna wireless passive strain sensor for use in a harsh environment. A patch antenna is terminated with a SAW delay line; the backscattered signal from the antenna can be distinguished from environmental reflections by a time delay. The patch antenna acts as both a usual device for receiving and radiating electromagnetic wave and a strain sensing device, and the SAW device behaves like a temperature indicator and a delay line reflector. Using the interdigital transducer (IDT) on SAW, the received electromagnetic waves could be translated into surface acoustic waves. After the reflection by several reflective gratings on SAW device, a set of reflected electromagnetic signals with different delay times could be generated. Since the delay time of the reflector changes with the temperature, it could be used as the temperature indicator. When preliminary data for the antenna material and the temperature-frequency characteristic are given, temperature fluctuation can be compensated. The high linearity of the frequency shift with temperature makes it possible to achieve a high accuracy for strain sensing. Simulation results and experimental results show that the presented antenna sensor could compensate for the temperature change; therefore, the strain sensing accuracy could be improved.

\section{Integrated SAW-Based Antenna Sensor Operation Principle}

The integrated SAW-based antenna sensor consists of two parts: a patch antenna which is bonded or embedded to a flexible structure and a SAW delay line which includes an IDT and several reflective gratings. The patch antenna is designed based on a quarter-wave rectangular patch antenna (folded-patch) topology [20]. The topology is chosen for its good radiation performance on top of metallic objects and $50 \%$ size reduction compared to regular patch antennas. The antenna structure is shown in Figure 1(a), and the resonant frequency, $f_{0}$, can be estimated as follows:

$$
f_{0}=\frac{c}{4(L+\Delta L) \sqrt{\varepsilon_{r}}},
$$

where $c$ is the speed of the light, $L$ is the physical length of the copper cladding on the antenna, and $\Delta L$ is the physical length deformation caused by the enforced strain, when the strain level is zero, $\Delta L=0 . \varepsilon_{r}$ is the effective dielectric constant of the antenna substrate.

The linear relationship between physical length and resonant frequency indicates that strain information can be derived by measuring the shift of the resonant frequency. This relationship could be used as the strain sensing mechanism, which is illustrated in Figure 1(b).

The power transmission in the passive wireless SAWbased antenna sensor system is shown in Figure 2. The interrogator transmits radio frequency signals, which are remotely received by the patch antenna. If the strain is enforced on the patch antenna, the resonant frequency of the backscattered signal will be changed accordingly.

The patch antenna is connected to a passive SAW delay line at the feed point. Firstly, the electromagnetic signal will enter the IDT and transformed into surface acoustic wave, which propagates along the surface of the SAW device. Secondly, after reflecting on several reflective gratings, a type of reflected pulses with different delay times will be generated. Typical delay time of the SAW device is $1 \sim 5$ microseconds.

With metal package, the response of the SAW device is just a function of temperature, not a function of strain. By analyzing the fluctuations of adjacent pulse delays, the temperature can be extracted. At a given temperature, the strain-resonant frequency curve is determined, so the strain could be extracted by analyzing the trivial change of the resonant frequency.

\section{Multiphysical Coupling Model of Integrated Antenna Sensors}

3.1. Strain and Temperature Sensing Using the Resonant Frequency Shift of the Patch Antenna. The resonant frequencies of the patch antenna are dependent on the dielectric constant of the substrate and the physical dimensions of its radiation patch [21]. 


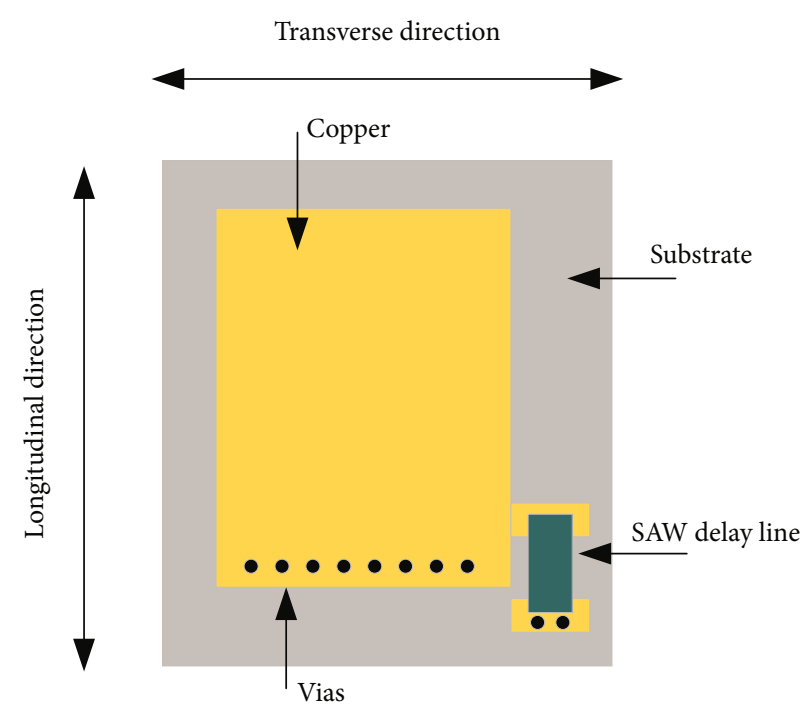

(a)

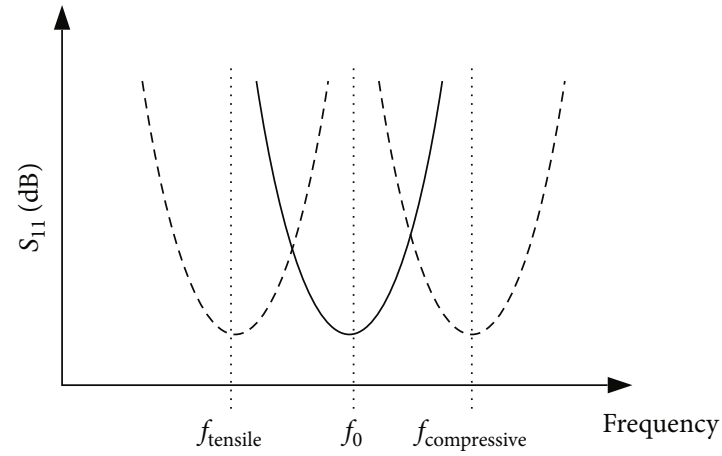

(b)

FIGURE 1: Sensing mechanism of a patch antenna sensor.

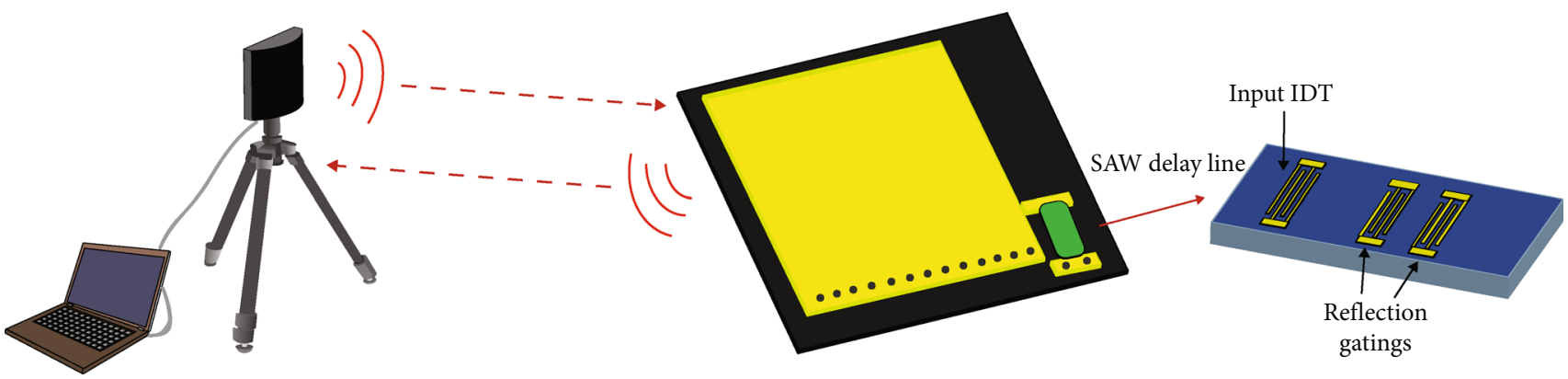

Figure 2: Power transmission in the passive wireless SAW-based antenna sensor system.

The resonant frequency shift $\delta f$ can then be obtained from changes in dielectric constant $\varepsilon_{r}$ and the radiation patch length $L$ as

$$
\delta f=\frac{\partial f}{\partial \varepsilon_{r}} \delta \varepsilon_{r}+\frac{\partial f}{\partial L} \delta L,
$$

where $\delta \varepsilon_{r}$ is the change in dielectric constant $\varepsilon_{r}$ and $\delta L$ is the change in the patch length. Both of these parameters are temperature-dependent.

Defining the ratio of $\delta \varepsilon_{r}$ to $\varepsilon_{r}$ as a relative change in dielectric constant, which is proportional to temperature change

$$
\frac{\delta \varepsilon_{r}}{\varepsilon_{r}}=\alpha_{\varepsilon} \delta T,
$$

where $\alpha_{\varepsilon}$ is the thermal coefficient of dielectric constant $\left(\mathrm{TCD}_{\mathrm{k}}\right)$ of the substrate and $\delta T$ is the temperature change.
Defining the ratio of $\delta L$ to $L$ as a relative change in the patch length, which is a function of both temperature change and the strain

$$
\frac{\delta L}{L}=\alpha_{t} \delta T+\varepsilon,
$$

where $\alpha_{\mathrm{t}}$ is the coefficient of thermal expansion (CTE) and $\varepsilon$ is the strain. Combining equations (1), (2), (3), and (4), the relationship between resonance frequency and strain/temperature could be obtained. When the temperature is given, strain information could be directly derived from the resonant frequency shift.

3.2. Thermal Effect Analysis of SAW Devices. In SAW devices, an electrical signal could be translated into an acoustic wave by an interdigital transducer. A coupled forward acoustic wave and a reverse propagating acoustic wave could be generated on the surface of SAW device. These coupled waves can be analyzed using coupling-of-modes (COM) equations [22]. 


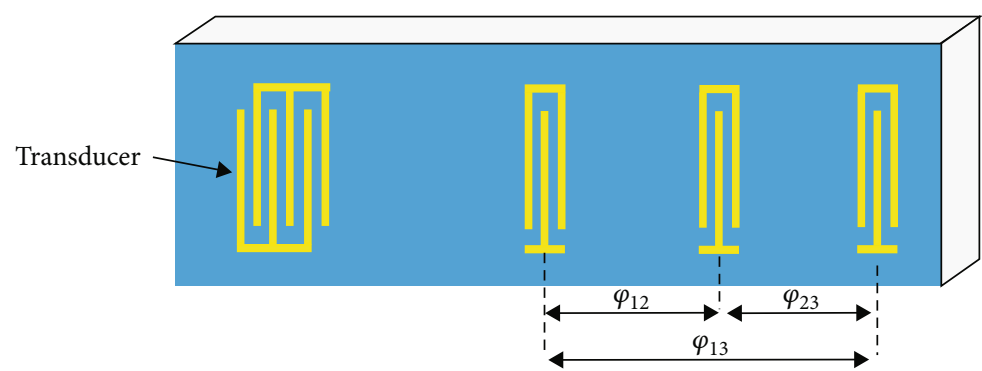

FIgURE 3: The structure of the SAW delay line and temperature sensor node.

Temperature fluctuation will change the wave velocity $v$ of SAW device by changing material stiffness. The temperature of coefficient delay (TCD) of a SAW device is expressed as follows:

$$
\mathrm{TCD}=\frac{1}{\tau} \frac{d \tau}{d T}
$$

where $\tau$ is the delay time of the reflector and $T$ is the current temperature.

The TCDs of SAW devices with different substrate materials are dissimilar. For example, SAW devices with lithium niobate substrate have a relative high TCD $\left(>90 \mathrm{ppm} /{ }^{\circ} \mathrm{C}\right)$, so the time delay will change significantly with the temperature variant.

A SAW sensor with three reflectors is shown in Figure 3. Generally, the phase difference between reflectors could eliminate the nonideal factors and high accuracy of time delay evaluation could be achieved. The mathematical expression is

$$
\varphi_{i j}=\varphi_{j}-\varphi_{i}=2 \pi f_{c}\left(\tau_{j}-\tau_{i}\right)=2 \pi f_{c} \tau_{i j} \quad(i, j=1,2,3, i \neq j),
$$

where $\varphi_{j}$ is the phase of the $j$ th reflector, $\varphi_{i}$ is the phase of the $i$ th reflector, $\varphi_{i j}$ is the phase difference between the $i$ th reflector and the $j$ th reflector, $f_{c}$ is the frequency of the surface acoustic wave, $\tau_{j}$ is the time delay of the $j$ th reflector, $\tau_{i}$ is the time delay of the $i$ th reflector, and $\tau_{i j}$ is the time difference between the $i$ th and the $j$ th reflectors. When the temperature varies, the phase difference $\varphi_{i j}$ will be changed accordingly as follows:

$$
\varphi_{i j}=\varphi_{i j}^{r}+\Delta \varphi_{i j}=\varphi_{i j}^{r}\left[1+S\left(T-T_{0}\right)\right]
$$

where $\varphi_{i j}^{r}$ is the phase difference under the condition of the reference; $\Delta \varphi_{i j}$ is the variation of phase difference which could be calculated from the measured data; $T$ is the temperature under test; $T_{0}$ is the temperature under the condition of the reference; and $S$ is the sensitivity of the SAW device, which is mainly determined by the substrate material of the SAW structure.

3.3. Local Field-Coupling Model Methods. The response of the integrated antenna sensor is related to the mutual coupling of several fields: structural displacement field, electromagnetic field, and temperature field, as illustrated in Figure 4.

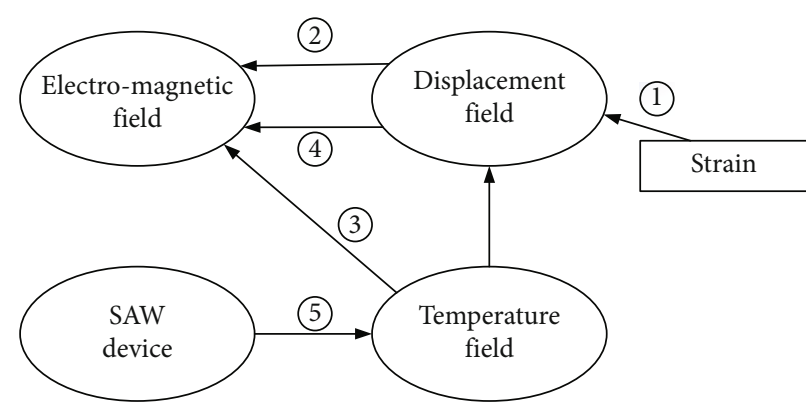

FIgURE 4: Multifield coupling of the integrated antenna sensor.

(1) Strain enforced on the patch antenna will lead to structure deformation, which could affect structural displacement field

(2) The structural displacement on the patch antenna will directly affect electromagnetic field

(3) Dielectric constant of the substrate on patch antenna is related to the temperature field. When the temperature alters, the dielectric constant will change accordingly, and finally leads to electromagnetic field variation

(4) Temperature field variation leads to displacement field fluctuation, and finally affects electromagnetic field

(5) Temperature field variation affects response of SAW device, and finally leads to electromagnetic field variation

The temperature could be extracted by the response of the SAW device in time domain, which is not convenient if the SAW device and patch antenna are modeled as a whole. For this reason, the integrated model is replaced by several local field-coupling models to approximately achieve multiphysical coupling as illustrated in Figure 5:

(1) Coupling-of-modes modeling of SAW device was conducted to extract the temperature and determine the output of admittance matrix parameters

(2) The displacement field of the patch antenna will changed with the temperature fluctuation and the enforced strain. The patch antenna is modeled in COMSOL, and the output of displacement parameters could be obtained 


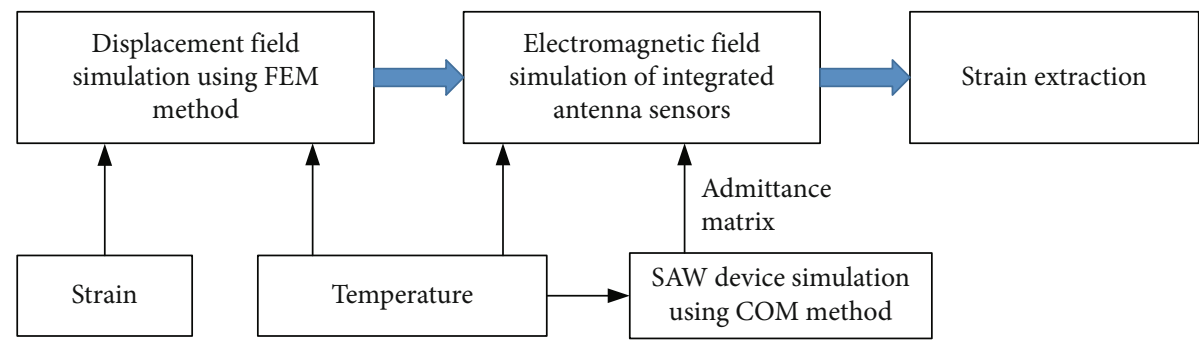

FIGURE 5: Antenna sensor multiphysical coupling simulation flow chart.

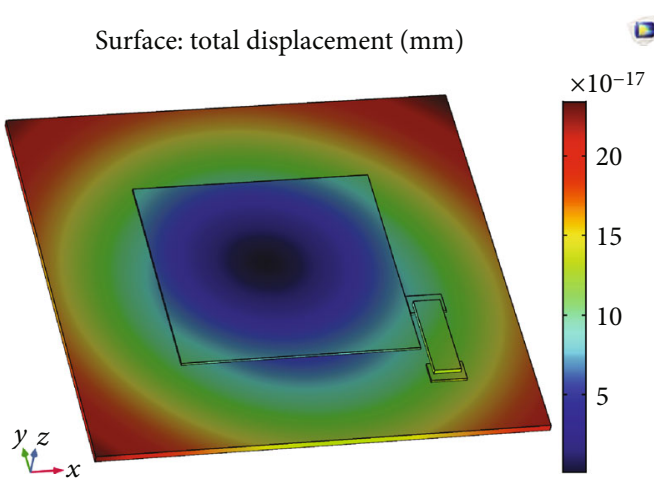

(a)

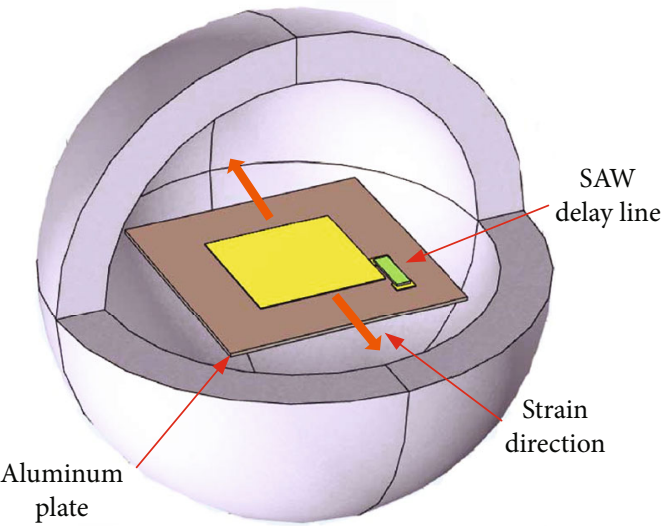

(b)

Figure 6: The integrated antenna sensor simulation in COMSOL. (a) Thermal expansion simulation and (b) tensile strain and frequency simulation.

(3) The outputs of the first two steps could be used as inputs for the integrated antenna sensor modeling. At the feed point of the patch antenna, the admittance matrix of SAW device is loaded to take the influence of SAW device into account. The patch size should be modified according to the displacement parameters. Besides, the dielectric constant of substrate is influenced by temperature variation

After the above three steps, the response of antenna sensor in frequency domain could be obtained and the resonant frequency could be extracted.

Since the temperature information has been extracted by SAW response, and the resonant frequency of patch antenna is linear with temperature and strain, the strain information can be extracted accurately with temperature compensation.

3.4. Strain Extraction Using Frequency Response of Integrated Antenna Sensors. In order to evaluate the deformation of the antenna patch under the influence of strain and temperature, the integrated antenna sensor is modeled in COMSOL and a two-step simulation is made.

First, a coupled thermal-structural analysis is performed to obtain the thermal expansion at various temperatures, as illustrated in Figure 6(a). Then, the deformation of the patch is exported as a three-dimensional structural file, which could be used as an import file to perform the mechanical analysis under various strains. The antenna is mounted on an aluminum specimen. Force is applied to the two ends of the aluminum specimen to generate strain.

The material of the patch is set to copper, the substrate material is set to Rogers RT/duroid ${ }^{\circledR} 5880$ with dielectric constant $\left(\varepsilon_{r}=2.2\right)$ and low loss tangent of 0.0009 . The thickness of the substrate is set to $0.254 \mathrm{~mm}$.

The deformation of the structure and the material parameters are used for modeling in COMSOL and simulated using FEM solver in frequency domain, as shown in Figure 6(b). The scattering parameter $S_{11}$ could be calculated as an output, which could indicate the corresponding resonance frequency. The simulated results of $S_{11}$ at different strain levels and temperatures are plotted in Figure 7.

The following trends could be seen from Figure 7: (1) at a given temperature, the resonance frequency of the antenna sensor will decrease due to the increasing strain and (2) when the strain level is fixed, the resonance frequency will change with the temperature fluctuation. When the temperature increases from $0^{\circ} \mathrm{C}$ to $60^{\circ} \mathrm{C}$ (see Figures $7(\mathrm{a})-7(\mathrm{~d})$ ), the resonance frequency of the integrated antenna sensor increases accordingly. This is because the $\mathrm{TCD}_{\mathrm{k}}$ factor of Rogers RT/duroid 5880 is negative, so the dielectric constant will be dropped due to the increasing temperature. A smaller dielectric constant will lead to a larger resonance frequency of the antenna. 


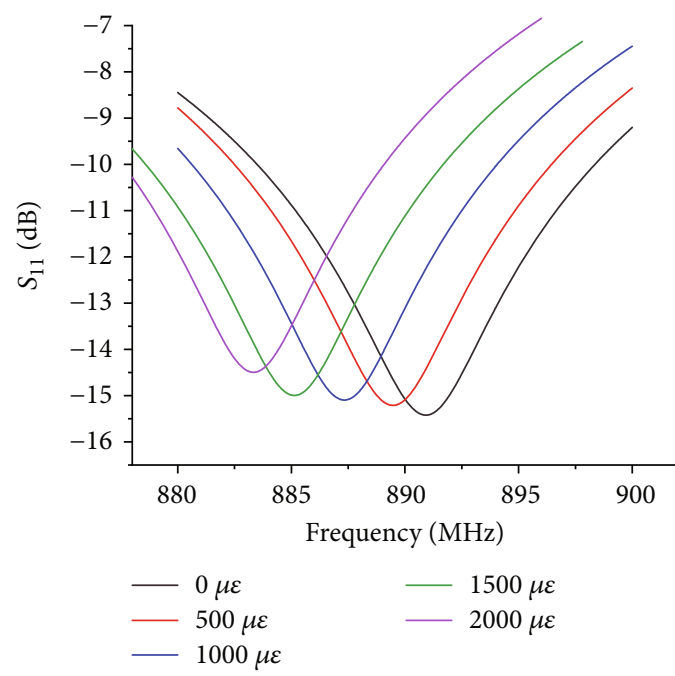

(a)

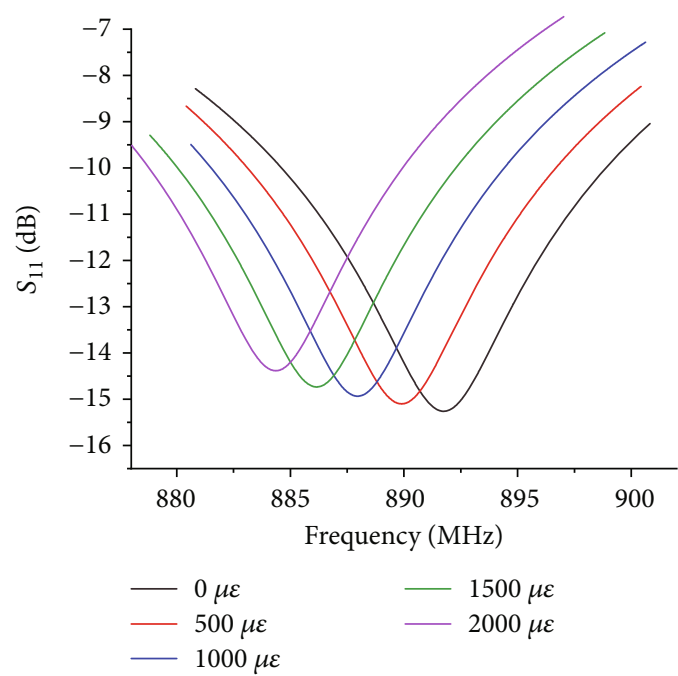

(c)

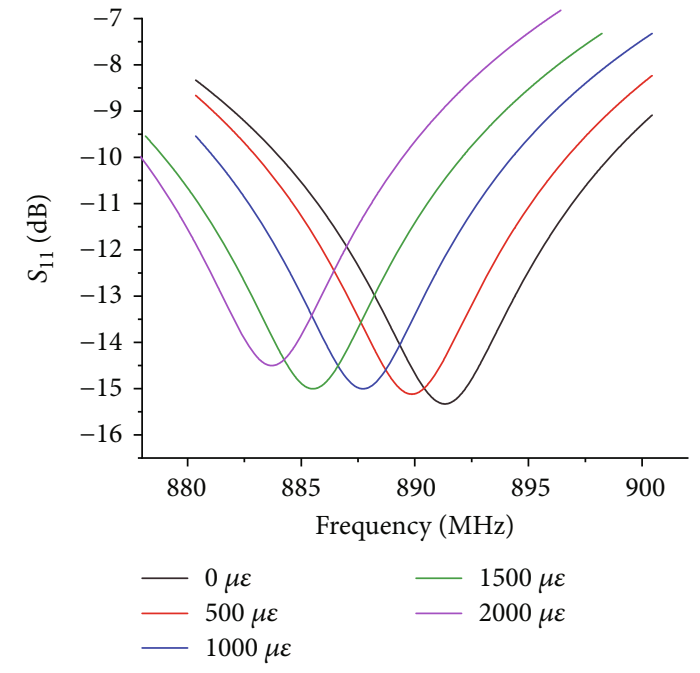

(b)

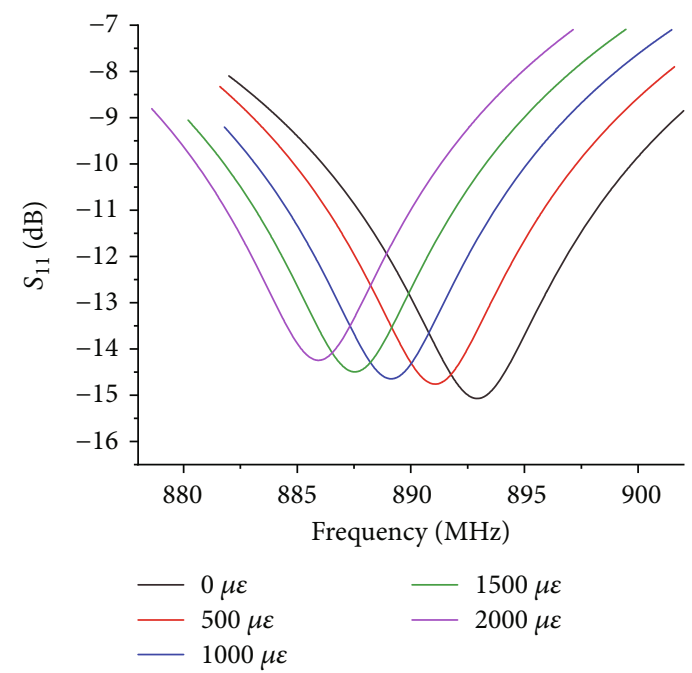

(d)

Figure 7: The simulated results of $S_{11}$ at different strain levels and temperatures. (a) $\mathrm{T}=0^{\circ} \mathrm{C}$; (b) $\mathrm{T}=20^{\circ} \mathrm{C}$; (c) $\mathrm{T}=40^{\circ} \mathrm{C}$; and $(\mathrm{d}) \mathrm{T}=60^{\circ} \mathrm{C}$.

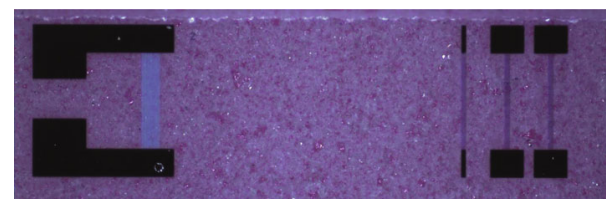

Figure 8: Photography of SAW delay line.

\section{Results and Discussion}

4.1. Temperature Extraction Using SAW Responses. A SAW device with $128^{\circ} \mathrm{YX}-\mathrm{LiNbO} 3$ substrate material is fabricated, which is shown in Figure 8.

The responses at different temperatures are measured. The SAW device is put into a temperature-controlled oven and the temperature ranges from $-50^{\circ} \mathrm{C}$ to $75^{\circ} \mathrm{C}$. The temperature information is extracted from the phase variation of the adjacent reflectors of the SAW device, which could be calculated from the time domain response. Measured amplitude and time delay of the reflectors in time domain are shown in Figure 9. Since the time delay of the surface acoustic wave corresponds to its phase, the difference between adjacent reflectors could be obtained without phase ambiguity.

The comparisons between the measured result and the simulated result are shown in Figure 10, where the red line is the theoretical temperature-phase line and the black dots are measured values at different temperatures. The temperaturephase relationship could be obtained from the measured results and has a good fit to the ideal line.

However, the measured results deviate slightly from the ideal linear relationship, probably because there is a tiny deviation between the actual measured temperature and the displayed temperature of the oven.

4.2. Instrumentation and Experimental Setup. The experimental setup is shown in Figure 11. The integrated antenna 


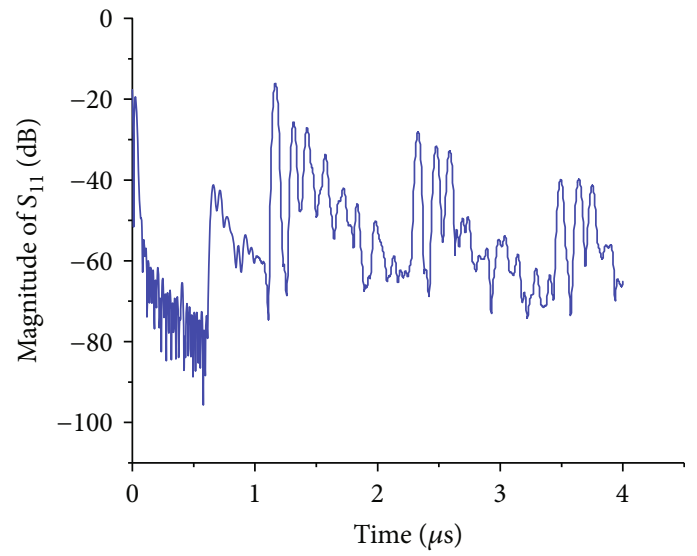

Figure 9: Measured amplitude and phase difference of the first and third reflectors in time domain.

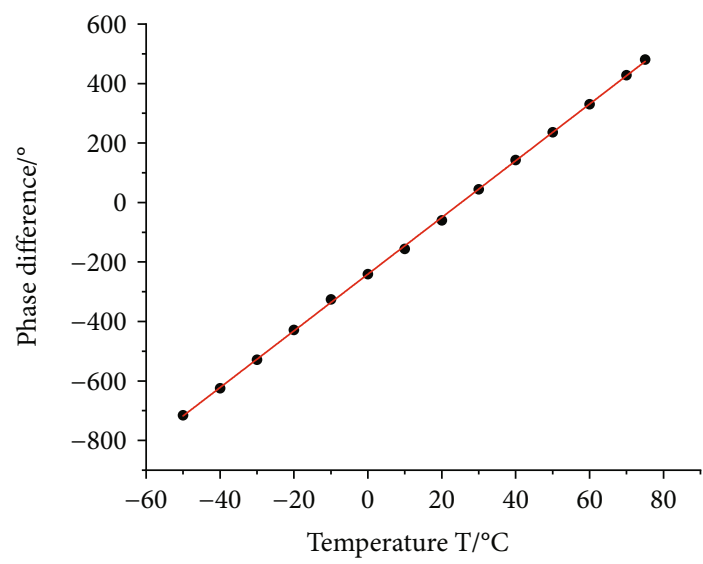

FIGURE 10: The relationship between the temperature and the phase difference.

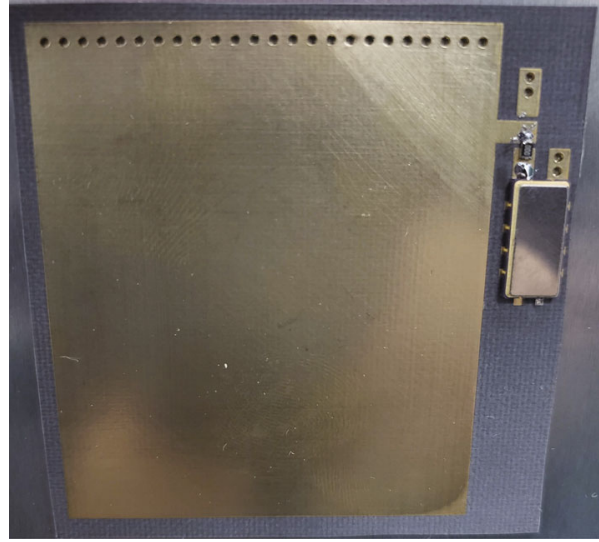

(a)

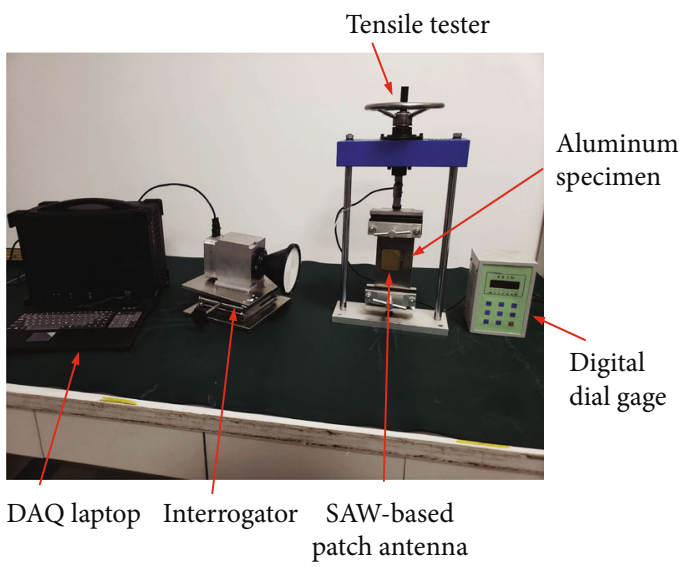

(b)

Figure 11: Experimental setup for the tensile tests. (a) Picture of the SAW-based patch antenna sensor. (b) Picture of the wireless strain sensing experiments.

sensor is fixed on a tensile tester. Aluminum plate which is the ground of the antenna is stretched on both sides to load strain on the integrated antenna sensor. By changing the stretching force, a series of strain values could be loaded. A hypothesis is given here that the stretching force and the strain on the integrated antenna sensor meet a linear 


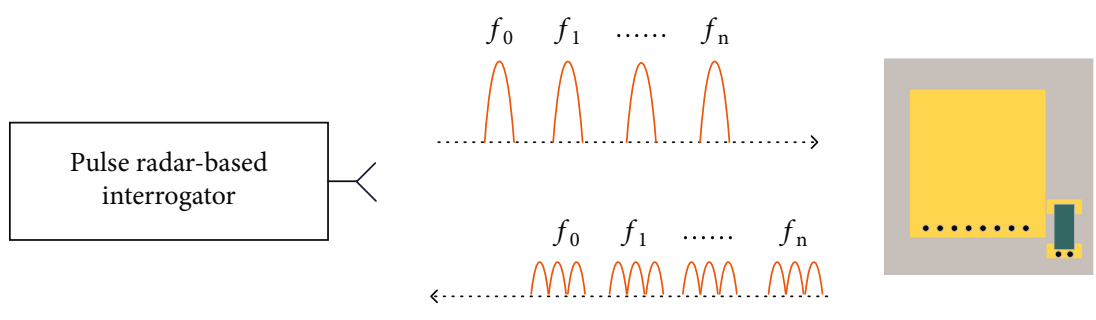

(a)

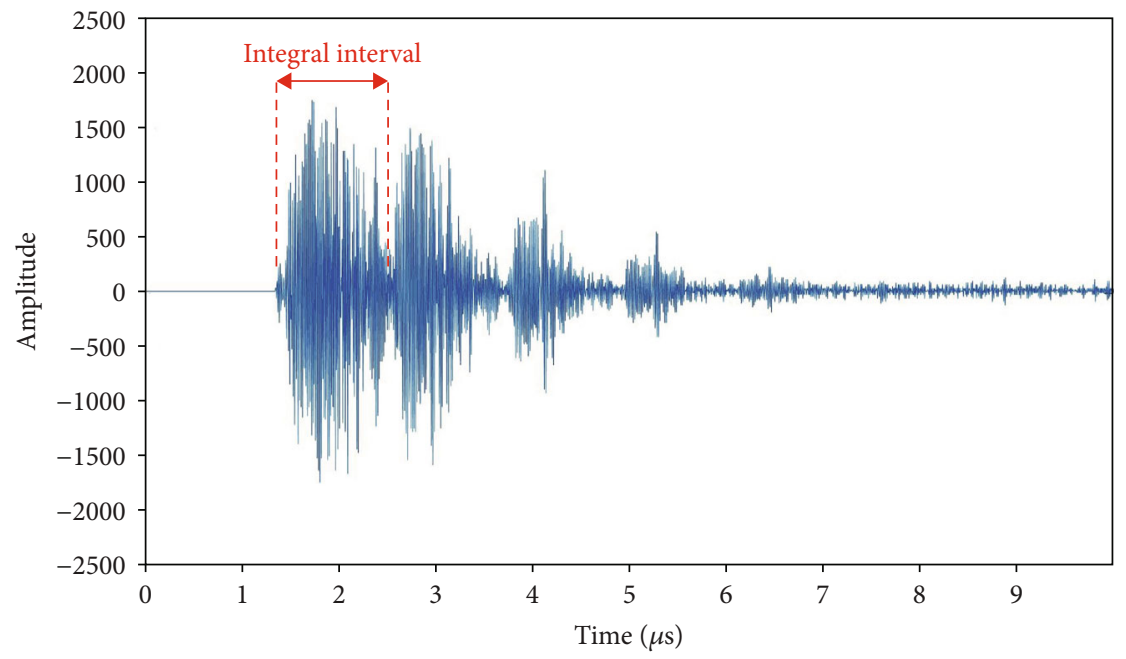

(b)

FIGURE 12: Measured spectrum of the antenna sensor's reflected signal. (a) Interrogation with a pulse radar-based reader. (b) Signal amplitude integral in order to restore the power curve.

relationship. A temperature oven is used to study the resonance frequency change caused by temperature fluctuation.

A series of single-toned signals which are transmitted by an interrogator and received by the integrated antenna sensor is shown in Figure 12(a). The signals are reflected by SAW reflectors; the echoed data has the same frequency with the transmitted signal and contains information of amplitude and phase shift. The temperature information is extracted from the phase shift difference of the adjacent echoed data. The amplitude of the echoed data is integrated during integral interval for each transmitted frequency value, as shown in Figure 12(b), to form the power spectrum density which is correlated to the resonance frequency.

The comparisons between measured and simulated relative resonance frequency variations $d f / f$ at different strains and temperatures are shown in Figure 13, where the triangular marks are measured results and the circular marks are simulated results. The measured results are highly close to the simulated ones. However, there are tiny deviations at several points, this is probably caused by the nonideal stretching error of the tensile tester.

Figure 13 compares the experimental and simulation results of strain/temperature and resonance frequency relationship for an integrated antenna sensor. When the strain is changed from 0 to $2000 \mu \varepsilon$ and the temperature is fixed, the resonance frequency will linearly decrease, and the total relative resonance frequency shift is up to $2000 \mathrm{ppm}$. When the temperature is changed from $20^{\circ} \mathrm{C}$ to $80^{\circ} \mathrm{C}$ and the strain

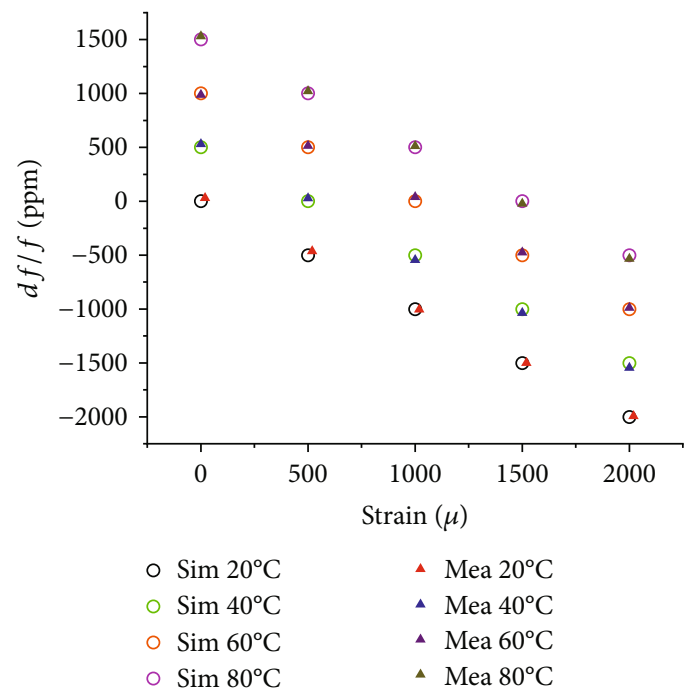

FIGURE 13: Comparison of experimental and simulation results of strain/temperature and resonance frequency relationship for an integrated antenna sensor.

is fixed, the resonance frequency will increase evenly, the total relative resonance frequency shift is about $1500 \mathrm{ppm}$.

4.3. Analysis. From Figure 13, the resonance frequency shift is proportional to the variation of the temperature and the strain. Since the temperature could be extracted separately 
by the phase difference of adjacent reflected echo data, the relationship between the resonance frequency shift and the strain could be built. On the other hand, a large time delay of the echoed data provided by the SAW device could separate the environmental clutter from the signal, which is beneficial for signal-to-noise ratio improvement.

Most of the previous antenna sensors proposed by others just testify the relationship between the resonance frequency and the strain; however, the effect of the temperature on the frequency response is not compensated. In this article, a novel integrated antenna sensor is proposed for wireless passive strain sensing of large strain range applications scenarios, such as wind turbine blades' structure health monitoring. Compared with previously presented antenna sensors, this proposed sensor could take the effect of temperature on the sensing result into account and a more accurate strain information could be extracted with temperature compensation for the first time.

\section{Conclusions}

A novel integrated antenna sensor is presented in this paper. The resonance frequency shift of the antenna sensor is linearly proportional to strain and temperature. The effect of temperature fluctuation on the resonance frequency shift is compensated by extracting temperature information from the phase difference of adjacent reflected echoed data, which is generated by the SAW reflective grating. Simulation and experimental results demonstrate the effectiveness of the strain sensing. This novel integrated antenna sensor provides more accurate passive (battery-free) wireless strain sensing for large-scale strain occasions than the previous proposed methods.

\section{Data Availability}

The measurement data and signal processing code used to support the findings of this study are available from the corresponding author upon request.

\section{Conflicts of Interest}

The authors declare that they have no conflicts of interest.

\section{References}

[1] H. Sohn, C. R. Farrar, F. M. Hemez et al., A Review of Structural Health Monitoring Literature: 1996-2001, Los Alamos National Laboratory, Los Alamos, USA, 2003.

[2] M. Bertolli, M. Donelli, A. Massa et al., "Computational methods for wireless structural health monitoring of cultural heritages," Journal of Physics: Conference Series, vol. 1131, 2018.

[3] Z. Chen, X. Zhou, X. Wang, L. Dong, and Y. Qian, "Deployment of a smart structural health monitoring system for long-span arch bridges: a review and a case study," Sensors, vol. 17, no. 9, p. 2151, 2017.

[4] L. Pantoli, M. Muttillo, G. Ferri et al., "Electronic system for structural and environmental building monitoring," in Convegno Nazionale Sensori, vol. 539, pp. 481-488, Springer, Cham, 2018.
[5] R. di Sante, "Fibre optic sensors for structural health monitoring of aircraft composite structures: recent advances and applications," Sensors, vol. 15, no. 8, pp. 18666-18713, 2015.

[6] L. Qiu, S. Yuan, X. Zhang, and Y. Wang, "A time reversal focusing based impact imaging method and its evaluation on complex composite structures," Smart Materials and Structures, vol. 20, no. 10, p. 105014, 2011.

[7] P. Wang, Y. Yan, G. Y. Tian, O. Bouzid, and Z. Ding, "Investigation of wireless sensor networks for structural health monitoring," Journal of Sensors, vol. 2012, 7 pages, 2012.

[8] O. M. Bouzid, G. Y. Tian, K. Cumanan, and D. Moore, "Structural health monitoring of wind turbine blades: acoustic source localization using wireless sensor networks," Journal of Sensors, vol. 2015, Article ID 139695, 11 pages, 2015.

[9] K. V. S. Rao, P. V. Nikitin, and S. F. Lam, “Antenna design for UHF RFID tags: a review and a practical application," IEEE Transactions on Antennas and Propagation, vol. 53, no. 12, pp. 3870-3876, 2005.

[10] X. Wang, O. Larsson, D. Platt et al., “An all-printed wireless humidity sensor label," Sensors and Actuators B: Chemical, vol. 166-167, pp. 556-561, 2012.

[11] G. Toth, K. Kordas, S. Myllymäki, and H. Jantunen, "Towards fully printed, antenna-based proximity sensors," Chinese Journal of Physics, vol. 50, no. 6, pp. 910-918, 2012.

[12] R. Bancroft, "Design of an integrated loop coupler and loop antenna for RFID applications," Microwave and Optical Technology Letters, vol. 51, no. 8, pp. 1830-1833, 2009.

[13] U. Tata, H. Huang, R. L. Carter, and J. C. Chiao, "Exploiting a patch antenna for strain measurements," Measurement Science and Technology, vol. 20, no. 1, p. 015201, 2009.

[14] S. Deshmukh and H. Huang, "Wireless interrogation of passive antenna sensors," Measurement Science and Technology, vol. 21, no. 3, p. 035201, 2010.

[15] X. Xu and H. Huang, "Battery-less wireless interrogation of microstrip patch antenna for strain sensing," Smart Materials and Structures, vol. 21, no. 12, p. 125007, 2012.

[16] C. Cho, X. Yi, D. Li, Y. Wang, and M. M. Tentzeris, "Passive wireless frequency doubling antenna sensor for strain and crack sensing," IEEE Sensors Journal, vol. 16, no. 14, pp. 5725-5733, 2016.

[17] X. Yi, C. Cho, J. Cooper, Y. Wang, M. M. Tentzeris, and R. T. Leon, "Passive wireless antenna sensor for strain and crack sensing-electromagnetic modeling, simulation, and testing," Smart Materials and Structures, vol. 22, no. 8, p. 085009, 2013.

[18] X. Yi, T. Wu, Y. Wang, R. T. Leon, M. M. Tentzeris, and G. Lantz, "Passive wireless smart-skin sensor using RFIDbased folded patch antennas," International Journal of Smart and Nano Materials, vol. 2, no. 1, pp. 22-38, 2011.

[19] F. M. Tchafa and H. Huang, "Microstrip patch antenna for simultaneous strain and temperature sensing," Smart Materials and Structures, vol. 27, no. 6, p. 065019, 2018.

[20] K. Finkenzeller, RFID Handbook, Wiley, New York, 2003.

[21] J. W. Sanders, J. Yao, and H. Huang, "Microstrip patch antenna temperature sensor," IEEE Sensors Journal, vol. 15, no. 9, pp. 5312-5319, 2015.

[22] V. Plessky and J. Koskela, "Coupling-of-modes analysis of SAW devices," International Journal of High Speed Electronics and Systems, vol. 10, no. 04, pp. 867-947, 2000. 


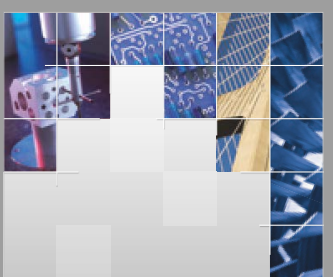

\section{Enfincering}
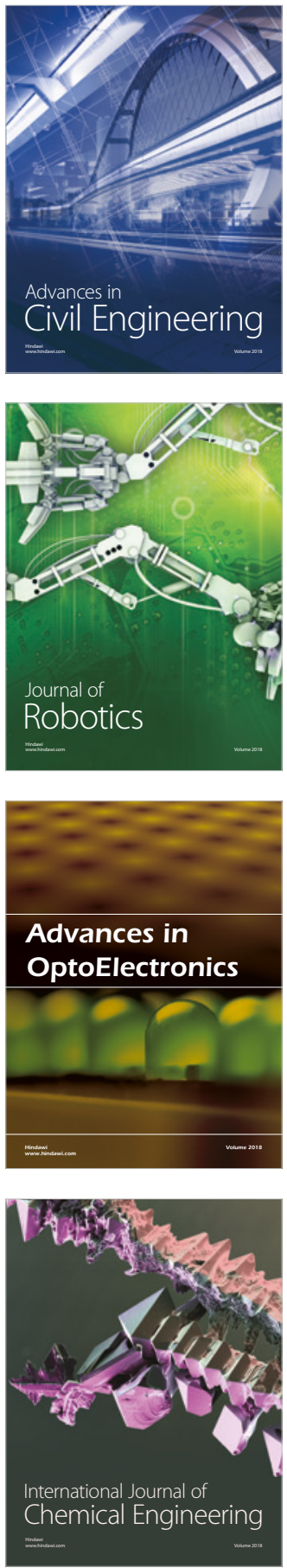

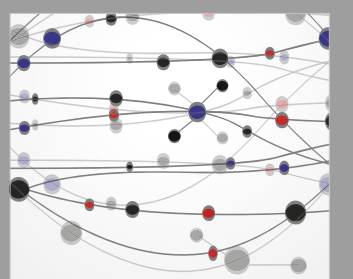

\section{Rotating \\ Machinery}

The Scientific World Journal

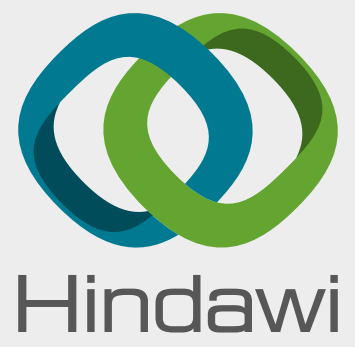

Submit your manuscripts at

www.hindawi.com
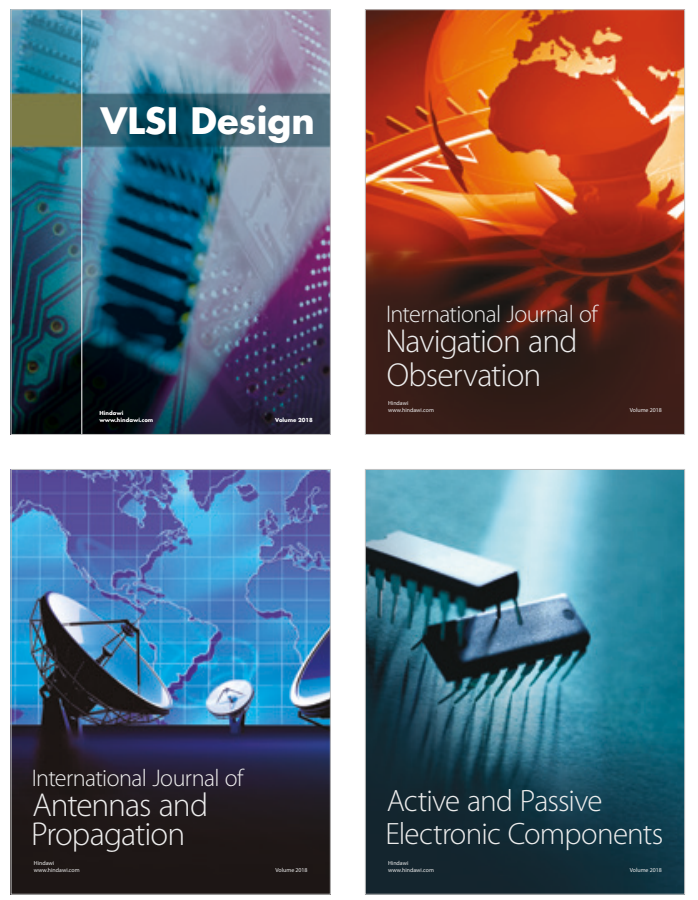
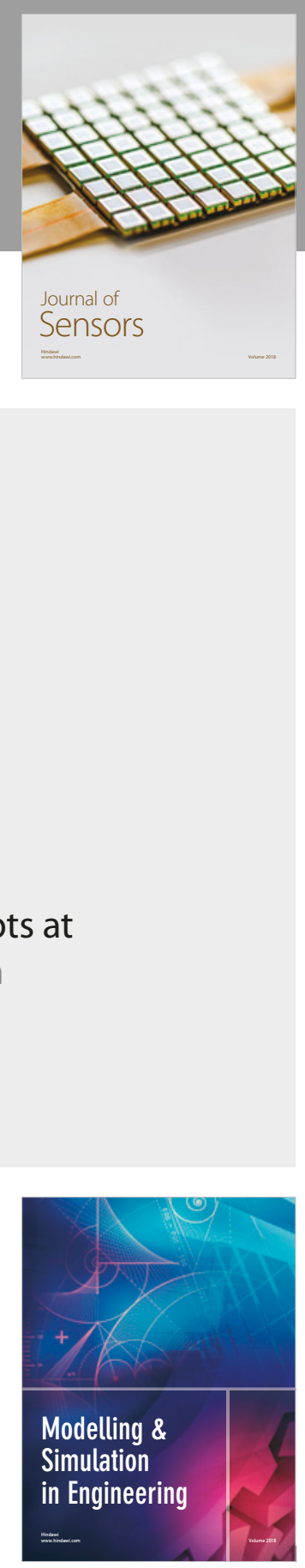

\section{Advances \\ Multimedia}
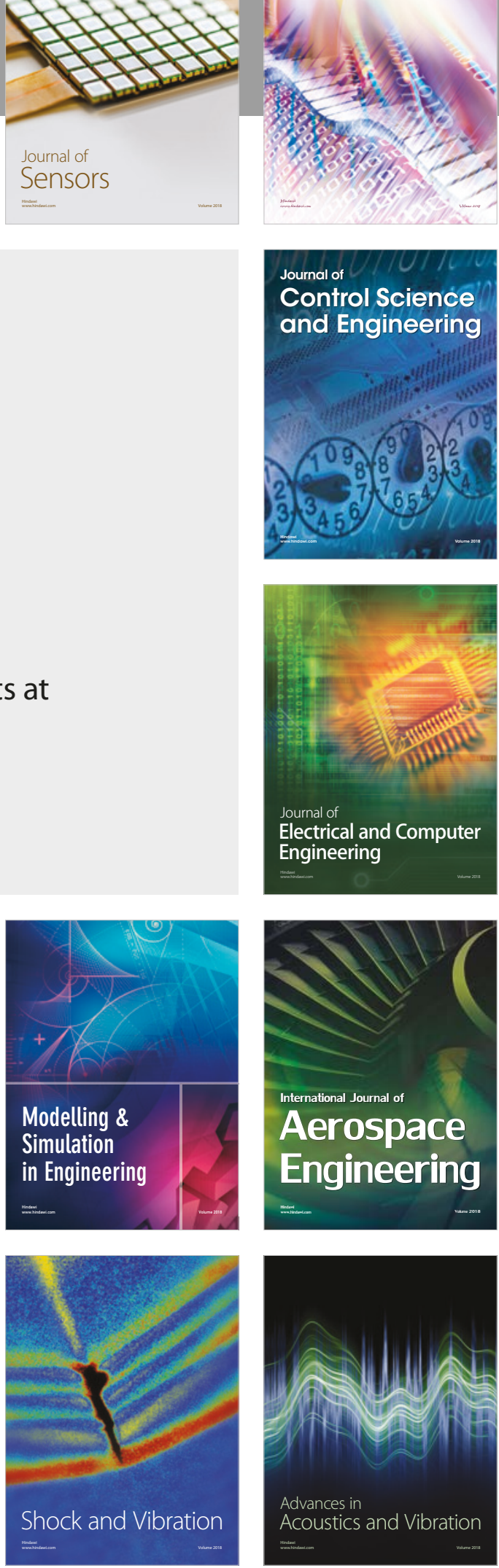